\title{
DEGREE BOUNDS FOR INVERSES OF POLYNOMIAL AUTOMORPHISMS
}

\author{
CHARLES CHING-AN CHENG, STUART SUI-SHENG WANG, AND JIE-TAI YU
}

(Communicated by Lance W. Small)

\begin{abstract}
It is known that if $k$ is a field and $\mathbf{F}: k\left[X_{1}, \ldots, X_{n}\right] \rightarrow$ $k\left[X_{1}, \ldots, X_{n}\right]$ is a polynomial automorphism, then $\operatorname{deg}\left(\mathbf{F}^{-1}\right) \leq(\operatorname{deg} \mathbf{F})^{n-1}$. We extend this result to the case where $k$ is a reduced ring. Furthermore, if $k$ is not a reduced ring, we show that for any integer $n \geq 1$ and any integer $\lambda \geq 0$ there exists a polynomial automorphism $\mathbf{F}$ such that $\operatorname{deg}\left(\mathbf{F}^{-1}\right)=$ $\lambda+(\operatorname{deg} \mathbf{F})^{n-1}$.
\end{abstract}

Let $k$ be a commutative ring with an identity element. Any $k$-algebra homomorphism $\mathbf{F}: k\left[X_{1}, \ldots, X_{n}\right] \rightarrow k\left[X_{1}, \ldots, X_{n}\right]$ is determined by the images of the $X_{i}$. Thus we denote $\mathbf{F}$ by $\left(F_{1}, \ldots, F_{n}\right)$, where $F_{i}=\mathbf{F}\left(X_{i}\right)$ for all $i$. The degree of $\mathbf{F}$ is defined to be the maximum of the total degrees of the $F_{i}$.

Theorem 1. Suppose $k$ is a field and F: $k\left[X_{1}, \ldots, X_{n}\right] \rightarrow k\left[X_{1}, \ldots, X_{n}\right]$ is a $k$-algebra automorphism. Then $\operatorname{deg}\left(\mathbf{F}^{-1}\right) \leq(\operatorname{deg} \mathbf{F})^{n-1}$.

Corollary 2. Suppose $k$ is an integral domain and $\mathbf{F}: k\left[X_{1}, \ldots, X_{n}\right] \rightarrow$ $k\left[X_{1}, \ldots, X_{n}\right]$ is a k-algebra automorphism. Then $\operatorname{deg}\left(\mathbf{F}^{-1}\right) \leq(\operatorname{deg} \mathbf{F})^{n-1}$.

Remark. The inequality in Theorem 1 was first conjectured by Wang in [3, Degree Conjecture 63, p. 491] for the case where $\operatorname{deg} F=2$. The general case was first proved by Gabber and published in [2, Corollary (1.4) and Theorem (1.5), pp. 292-293], using algebraic geometry. A more elementary proof was given by $\mathrm{Yu}$ in [4, Theorem 2].

In the following we shall show that reduced rings are the best possible rings in which this inequality holds for all polynomial automorphisms.

A reduced ring $k$ is a commutative ring whose nilradical ( $=$ intersection of all prime ideals) is zero or, equivalently [1, Propositions 1.7 and 1.8, p. 5], a commutative ring with no nonzero nilpotent elements. Given a prime ideal $\mathfrak{p}$ of $k$, we denote the quotient ring $k / \mathfrak{p}$ by $\bar{k}$. For each polynomial $H=$ $\sum c_{i_{1}}, \ldots, i_{n} X_{1}^{i_{1}} \cdots X_{n}^{i_{n}}$ in $k\left[X_{1}, \ldots, X_{n}\right]$, let $\bar{H}=\sum\left(c_{i_{1}}, \ldots, i_{n}+\mathfrak{p}\right) X_{1}^{i_{1}} \cdots X_{n}^{i_{n}}$ be the induced polynomial in $\bar{k}\left[X_{1}, \ldots, X_{n}\right]$. Note that $\operatorname{deg} \bar{H} \leq \operatorname{deg} H$. Also

Received by the editors June 26, 1992.

1991 Mathematics Subject Classification. Primary 14E09, 13B25, 13 F20.

Key words and phrases. Automorphism, degree, inverse, polynomial ring, reduced ring, nilpotent element, nilradical. 
note that each $k$-algebra endomorphism $\mathbf{F}=\left(F_{1}, \ldots, F_{n}\right): k\left[X_{1}, \ldots, X_{n}\right] \rightarrow$ $k\left[X_{1}, \ldots, X_{n}\right]$ induces a $\bar{k}$-algebra endomorphism $\overline{\mathbf{F}}=\left(\bar{F}_{1}, \ldots, \bar{F}_{n}\right)$ : $\bar{k}\left[X_{1}, \ldots, X_{n}\right] \rightarrow \bar{k}\left[X_{1}, \ldots, X_{n}\right]$. In this case $\operatorname{deg} \overline{\mathbf{F}} \leq \operatorname{deg} \mathbf{F}$.

Theorem 3. Suppose $k$ is a reduced ring and $\mathbf{F}: k\left[X_{1}, \ldots, X_{n}\right] \rightarrow$ $k\left[X_{1}, \ldots, X_{n}\right]$ is a k-algebra automorphism. Then $\operatorname{deg}\left(\mathbf{F}^{-1}\right) \leq(\operatorname{deg} \mathbf{F})^{n-1}$.

Proof. Let $\mathbf{F}^{-1}=\mathbf{G}=\left(G_{1}, \ldots, G_{n}\right)$ be the inverse of $F$. Let $1 \leq i \leq n$ be such that $\operatorname{deg} G_{i}=\operatorname{deg} \mathbf{G}$. Then there exists a prime ideal $\mathfrak{p}$ of $k$ such that $\operatorname{deg} \bar{G}_{i}=\operatorname{deg} G_{i}$. Otherwise, $\operatorname{deg} \bar{G}_{i}<\operatorname{deg} G_{i}$ for all prime ideals of $k$ and so the nonzero coefficients of the highest degree terms of $G_{i}$ are in the nilradical of $k$. Since $k$ is reduced, this contradicts the fact that the nilradical of $k$ is zero. By Corollary $2, \operatorname{deg} \overline{\mathbf{G}} \leq(\operatorname{deg} \overline{\mathbf{F}})^{n-1}$. Thus,

$$
\operatorname{deg} \mathbf{G}=\operatorname{deg} \overline{\mathbf{G}} \leq(\operatorname{deg} \overline{\mathbf{F}})^{n-1} \leq(\operatorname{deg} \mathbf{F})^{n-1} .
$$

Theorem 4. Suppose $k$ is not a reduced ring. Then, for any positive integer $n$ and any nonnegative integer $\lambda$, there exists a $k$-algebra automorphism $\mathbf{F}: k\left[X_{1}\right.$, $\left.\ldots, X_{n}\right] \rightarrow k\left[X_{1}, \ldots, X_{n}\right]$ such that $\operatorname{deg}\left(\mathbf{F}^{-1}\right)=\lambda+(\operatorname{deg} \mathbf{F})^{n-1}$.

Proof. Since $k$ is not reduced, there exists a nonzero element $v \in k$ such that $v^{2}=0$. (If $u$ is a nonzero nilpotent element in $k$ such that $u^{d+1}=0$, but $u^{d} \neq 0$ for some positive integer $d$, take $v=u^{d}$.) Thus, for any integer $m \geq 0$ and $A, B$ in $k\left[X_{1}, \ldots, X_{n}\right]$, as a direct consequence of the binomial theorem, we have that

$$
(A \pm v B)^{m}=A^{m} \pm m v A^{m-1} B .
$$

Now choose a positive integer $e$ such that

(i) $e \geq \lambda+1$, and

(ii) $e^{n-1} v \neq 0$ (so that none of $v, e v, e^{2} v, \ldots, e^{n-1} v$ is zero).

This can be done as follows. The annihilator of $v$ in $\mathbb{Z}$, i.e., $\{l \in \mathbb{Z} \mid l v=0\}$, is a principal ideal generated by a nonnegative integer $\mu$. Take

$$
e= \begin{cases}\lambda+1, & \text { if } \mu=0 \\ \lambda \mu+1, & \text { if } \mu \geq 1\end{cases}
$$

Define $\mathbf{F}=\left(F_{1}, \ldots, F_{n}\right): k\left[X_{1}, \ldots, X_{n}\right] \rightarrow k\left[X_{1}, \ldots, X_{n}\right]$ by

$$
F_{1}=X_{1}-v X_{1}^{\lambda+1}, \quad F_{i}=X_{i}-X_{i-1}^{e} \quad \text { for } i=2, \ldots, n .
$$

We next show that the inverse of $\mathbf{F}$ is $\mathbf{G}=\left(G_{1}, \ldots, G_{n}\right)$ where

$$
G_{1}=X_{1}+v X_{1}^{\lambda+1}, \quad G_{i}=X_{i}+G_{i-1}^{e} \text { for } i=2, \ldots, n .
$$

Note that

$$
\begin{aligned}
F_{1}\left(G_{1}, \ldots, G_{n}\right) & =G_{1}-v G_{1}^{\lambda+1} \quad\left(\text { by the definition of } F_{1}\right) \\
& =X_{1}+v X_{1}^{\lambda+1}-v\left(X_{1}+v X_{1}^{\lambda+1}\right)^{\lambda+1} \quad\left(\text { by the definition of } G_{1}\right) \\
& =X_{1}+v X_{1}^{\lambda+1}-v\left(X_{1}^{\lambda+1}+(\lambda+1) v X_{1}^{2 \lambda+1}\right) \quad(\text { by }(1)) \\
& =X_{1} .
\end{aligned}
$$


Similarly $G_{1}\left(F_{1}, \ldots, F_{n}\right)=X_{1}$. For $2 \leq i \leq n$,

$$
\begin{aligned}
F_{i}\left(G_{1}, \ldots, G_{n}\right) & =G_{i}-G_{i-1}^{e} \quad\left(\text { by the definition of } F_{i}\right) \\
& =X_{i} \quad\left(\text { by the definition of } G_{i}\right), \\
G_{i}\left(F_{1}, \ldots, F_{n}\right) & =F_{i}+G_{i-1}\left(F_{1}, \ldots, F_{n}\right)^{e} \quad\left(\text { by the definition of } G_{i}\right) \\
& =F_{i}+X_{i-1}^{e} \quad(\text { by induction on } i) \\
& =X_{i} \quad\left(\text { by the definition of } F_{i}\right) .
\end{aligned}
$$

Therefore, $\mathbf{F}$ and $\mathbf{G}$ are inverses of each other.

We next compute $\operatorname{deg} \mathbf{G}$. If $e \geq 2$, then using (1) we can prove by induction on $i$ that

$$
\begin{aligned}
G_{i}= & \left(X_{1}^{e^{i-1}}+\text { lower degree terms }\right) \\
& +v\left(e^{i-1} X_{1}^{\lambda+e^{i-1}}+\text { lower degree terms }\right)
\end{aligned}
$$

where "lower degree terms" involves no $v$. Hence, $\operatorname{deg} G_{i}=\lambda+e^{i-1}$ by condition (ii). If $e=1$, then we can prove by induction on $i$ that $G_{i}=$ $\left(X_{1}+\cdots+X_{i}\right)+v X_{1}^{\lambda+1}$. Hence, $\operatorname{deg} G_{i}=\lambda+1=\lambda+e^{i-1}$ in this case. Therefore,

$$
\operatorname{deg} \mathbf{G}=\max _{1 \leq i \leq n}\left\{\operatorname{deg} G_{i}\right\}=\max _{1 \leq i \leq n}\left\{\lambda+e^{i-1}\right\}=\lambda+e^{n-1},
$$

i.e., $\operatorname{deg} \mathbf{G}=\lambda+(\operatorname{deg} \mathbf{F})^{n-1}$, since $\operatorname{deg} \mathbf{F}=e$ by condition (i).

\section{REFERENCES}

1. M. F. Atiyah and I. G. Macdonald, Introduction to commutative algebra, Addison-Wesley, Reading, MA, 1969. (MR 39\#4129)

2. H. Bass, E. Connell, and D. Wright, The Jacobian conjecture. Reduction of degree and formal expansion of the inverse, Bull. Amer. Math. Soc. (N.S.) 7 (1982), 287-330. (MR 83k:14028. Zbl.539.13012)

3. S. S.-S. Wang, A Jacobian criterion for separability, J. Algebra 65 (1980), 453-494. (MR 83e:14010 Zbl.471.13005)

4. J.-T. Yu, Degree bounds of minimal polynomials and polynomial automorphisms, J. Pure Appl. Algebra (to appear).

(C. C.-A. Cheng and S. S.-S. Wang) Department of Mathematical Sciences, Oakland UNIVERSITY, ROCHESTER, MICHIGAN 48309-4401

E-mail address, C. C.-A. Cheng: cheng@vela.acs.oakland.edu

E-mail address, S. S.-S. Wang: swang@vela.acs.oakland.edu

(J.-T. Yu) Department of Mathematics, University of Notre Dame, Notre Dame, IndANA 46556-5683

E-mail address: jyu@hilbert.helios.nd.edu 